\title{
Obtaining Kohn-Sham potential without taking the functional derivative
}

\author{
MANOJ K HARBOLA* and K D SEN ${ }^{\dagger}$ \\ Department of Physics, Indian Institute of Technology, Kanpur 208 016, India \\ 'School of Chemistry, University of Hyderabad, Hyderabad 500 046, India
}

\begin{abstract}
Over the past decade and a half, many new accurate density functionals, based on the generalized gradient approximation, have been proposed, and they give energies close to chemical accuracy. However, accuracy of the energy functional does not guarantee that its functional derivative, which gives the corresponding potential, is also accurate all over space. For example, although the Becke88 exchange-energy functional gives very good exchange energies, its functional derivative goes as $-\frac{1}{2}$ in comparison to the correct $-\frac{1}{r}$ for $r \rightarrow \infty$, where $\boldsymbol{r}$ is the distance of the electron from a finite system. On the other hand, accuracy of the potential is of prime importance if one is interested in properties other than the total energy; properties such as optical response depend crucially on the potential in the outer regions of a system. In this paper we present a different approach, based on the ideas of Harbola and Sahni, to obtain the potential directly from the energy density of a given approximation, without taking recourse to the functional derivative route. This leads to a potential that is as accurate as the functional itself. We demonstrate the accuracy of our approach by presenting some results obtained from the Becke88 functional.
\end{abstract}

Keywords. Kohn-Sham potential; density functionals; generalized gradient approximation.

\section{Introduction}

Hohenberg-Kohn-Sham density-functional theory (DFT) (Parr and Yang 1989; Dreizler and Gross 1990) provides the basis of all modern-day electronic-structure calculations. As is well known, in this theory electron density plays the role of the basic variable. Thus the total energy is expressed in terms of the density as

$$
\begin{aligned}
& E[\boldsymbol{\rho}]=T_{\mathrm{s}}[\boldsymbol{\rho}]+\int v_{\mathrm{ext}}(\mathbf{r}) \boldsymbol{\rho}(\mathbf{r}) \mathrm{d} \mathbf{r} \\
& +\frac{1}{2} \iint \frac{\boldsymbol{\rho}(\mathbf{r}) \boldsymbol{\rho}\left(\mathbf{r}^{\prime}\right)}{\left|\mathbf{r}-\mathbf{r}^{\prime}\right|} \mathrm{d} \mathbf{r} \mathbf{r}^{\prime}+E_{\mathrm{xc}}[\boldsymbol{\rho}]
\end{aligned}
$$

Here $T_{\mathrm{s}}[\rho]$ is the kinetic energy of non-interacting electrons of density $\rho(\mathbf{r}), v_{\text {ext }}(\mathbf{r})$ the potential electrons are moving in, $\frac{1}{2} \iint \frac{\rho(\mathbf{r}) \rho\left(\mathbf{r}^{\prime}\right)}{\left|\mathbf{r}-\mathbf{r}^{\prime}\right|} \mathrm{d} \mathbf{r} \mathrm{d} \mathbf{r}^{\prime}$ the direct Coulomb energy of electrons and $E_{\mathrm{xc}}[\rho]$ their exchange-correlation energy. The density is obtained from the solutions $\phi(\mathbf{r})$ of the Kohn-Sham equation (all equations are written in atomic units)

$$
\begin{aligned}
{\left[-\frac{1}{2} \nabla^{2}\right.} & +v_{\mathrm{ext}}(\mathbf{r}) \\
& \left.+\int \frac{\rho\left(\mathbf{r}^{\prime}\right)}{\left|\mathbf{r}-\mathbf{r}^{\prime}\right|} \mathrm{d} \mathbf{r}^{\prime}+v_{\mathrm{xc}}(\mathbf{r})\right] \phi_{i}(\mathbf{r})=\varepsilon_{i} \phi_{i}(\mathbf{r}),
\end{aligned}
$$

\footnotetext{
*Author for correspondence
}

as

$$
\rho(\mathbf{r})=\sum_{i}\left|\phi_{i}(\mathbf{r})\right|^{2}
$$

The exchange-correlation potential, $v_{\mathrm{xc}}(\mathbf{r})$, in the KohnSham equation is obtained by taking the functional derivative $\frac{\delta E_{x c}[\rho]}{\delta \rho(\mathbf{r})}$ of the exchange-correlation energy functional, $E_{\mathrm{xc}}[\boldsymbol{\rho}]$. Since the inception of densityfunctional theory, the local-density-approximation (LDA) and the local-spin-density-approximation (LSDA) (Parr and Yang 1988; Dreizler and Gross 1990) have been the mainstay of most of the DFT calculations. These approximations are based on the exchange-correlation energies of homogeneous electron gas. Thus the exchange energy in the LDA is given as

$$
E_{\mathrm{x}}^{\mathrm{LDA}}=\int \varepsilon_{\mathrm{x}}(\mathbf{r}) \dot{\rho}(\mathbf{r}) \mathrm{d} \mathbf{r},
$$

where $\varepsilon_{\mathrm{X}}$ is the exchange energy per particle for a homogeneous electron gas, expressed in terms of the density $\rho(\mathbf{r})$ as

$$
\varepsilon_{\mathrm{x}}(\mathbf{r})=-\frac{3 k_{\mathrm{F}}(\mathbf{r})}{4 \pi}
$$

where $k_{\mathrm{F}}(\mathbf{r})=\left(3 \pi^{2} \rho(\mathbf{r})\right)^{\frac{1}{3}}$ is the local Fermi vector. Correlation energy in the LDA is also given by an expression similar to (4) above with the exchange-energy, $\varepsilon_{\mathrm{x}}$, per particle replaced by the correlation energy, $\varepsilon_{c}$, per particle. In the parametrization by Gunnarsson and Lundquist (1976), correlation energy per electron is given as 


$$
\varepsilon_{\mathrm{c}}(\mathbf{r})=-0 \cdot 0333\left[\left(1+x^{3}\right) \ln \left(1+\frac{1}{x}\right)+\frac{1}{2} x-x^{2}-\frac{1}{3}\right],
$$

where

$$
x=\frac{r_{\mathrm{s}}}{11 \cdot 4},
$$

and $r_{\mathrm{s}}=\left[\frac{3}{4 \pi}\left(\frac{1}{\rho}\right)\right]^{\frac{1}{3}}$ measures the radius of a sphere enclosing one electron. The corresponding exchange and correlation potentials are given as

$$
v_{\mathrm{x}}(\mathbf{r})=-\frac{k_{\mathrm{F}}(\mathbf{r})}{\pi}
$$

and

$$
v_{\mathrm{c}}(\mathbf{r})=-0 \cdot 0333 \ln \left(1+\frac{1}{x}\right) .
$$

Since the LDA is based on homogeneous electron gas results, it is expected to be accurate when the density is a slowly varying function. However, if the density has large inhomogeneity, the error in the LDA becomes relatively large. To incorporate the non-local dependence of the exchange-correlation energy on density, one then makes a Taylor series expansion-termed as the gradient expansion approximation or the GEA-of the energy in terms of the gradient of the density. Such a correction to the LDA was first proposed for the exchange energy by Herman et al (1969). The GEA functional up to the second-order in the density gradients is

$$
E_{\mathrm{x}}^{\mathrm{GEA}}[\rho]=E_{\mathrm{x}}^{\mathrm{LDA}}[\rho]+C_{\mathrm{x}} \int \frac{|\nabla \rho|^{2}}{\rho^{\frac{4}{3}}} \mathrm{~d} \mathbf{r}
$$

where $\quad C_{x}=\frac{7}{432 \pi\left(3 \pi^{2}\right)^{1 / 3}}$. The exchange-potential corresponding to the GEA functional is

$$
v_{\mathrm{x}}^{\mathrm{GEA}}(\mathbf{r})=v_{\mathrm{x}}^{\mathrm{LDA}}(\mathbf{r})+C_{\mathrm{x}}\left[\frac{4|\nabla \rho|^{2}}{3} \frac{\nabla^{2} \rho}{\rho^{\frac{7}{3}}}-2 \frac{\rho^{\frac{4}{3}}}{.} .\right.
$$

For exponentially decaying densities, this potential becomes singular at large distances so that in implementing it in the Kohn-Sham scheme, a damping term has to be introduced. This is one of the problems with the GEA. Other problems are related (Perdew 1985) to the Fermi hole corresponding to the GEA energy. The GEA has been further improved upon by rectifying these shortcomings to develop functionals known as the generalized-gradient approximation (GGA). These functionals have been shown to possess chemical accuracy. The most popular ones are the PW91 (Perdew and Wang 1986; Perdew 1991) and Becke88 (Becke 1988) functionals.

An important point that must be kept in mind in developing energy functionals is that their functional deriva- tives be easy to obtain so that the corresponding potential can be easily calculated. However, accuracy of a functional does not automatically guarantee that its functional derivative will also be accurate. For example, the Becke88 functional gives the exact exchange-energy density $-\frac{1}{2 r}$ asymptotically but its functional derivative goes (Engel et al 1992) as $-\frac{1}{2^{2}}$. Thus it cannot be expected to give properties-such as the highest-occupied orbital energy-which depend to a large extent on the behaviour of the potential in the outer regions of a system, correctly. Further, in atoms the functional derivative diverges (Engel et al 1992) near the nucleus as $-\frac{1}{r}$ as $r \rightarrow 0$, whereas the effective potential is finite at the nucleus (Umrigar and Gonze 1994; Qian and Sahni 2000). Due to this overestimate of the effective local potential close to the nucleus, electronic properties such as the electron-nuclear cusp condition, total s-electron density and the spin density at the nucleus are expected to be poorly represented. Motivated by these issues, a question that we ask is if it is possible to obtain the effective potential without taking the functional derivative of the energy functional. Such an approach is useful on two fronts: First, it frees one from fitting an energy functional to bring it to a manageable form so that its functional derivative could be obtained, and secondly by circumventing the functional derivative route to get the effective potential, it keeps the accuracy of the potential at the same level as that of the energy density. In the following we present such an approach for the exchange potential, based upon the ideas of Slater (1951) and Harbola and Sahni (1989). We therefore start with a description of the Slater potential, then introduce the idea of electric field due to the Fermi-Coulomb hole, give an exact prescription of the exact exchange-correlation potential in terms of this field and finally use this prescription to obtain an approximate exchange potential in terms of the exchange energy density. We employ this scheme with the Becke88 energy functional and present the results obtained for the energies and ionization potential of some closed shell atoms.

\section{Exchange-correlation potential from the Fermi-Coulomb hole}

To simplify the Hartree-Fock theory (Slater 1960), which has orbital-dependent non-local exchange-potentials, and make it computationally easy, Slater (1951) introduced the idea of obtaining a local exchange potential from the Fermi hole of an electron. Without going into the details of its derivation, which are well discussed (Harbola and Sahni 1993) in the literature, we give the form of the Slater potential here. It is given as

$$
V_{\text {Slater }}(\mathbf{r})=\int \frac{\rho_{\mathrm{x}}\left(\mathbf{r}, \mathbf{r}^{\prime}\right)}{\left|\mathbf{r}-\mathbf{r}^{\prime}\right|} \mathrm{d} \mathbf{r}^{\prime},
$$


where $\rho_{\mathrm{x}}\left(\mathbf{r}, \mathbf{r}^{\prime}\right)$ is the Fermi hole arising due to the Pauli exclusion principle. It is given in terms of the occupied orbitals of a system (for simplicity, we are considering here only the closed-shell case) as

$$
\rho_{\mathrm{x}}\left(\mathbf{r}, \mathbf{r}^{\prime}\right)=\frac{\left|\gamma\left(\mathbf{r}, \mathbf{r}^{\prime}\right)\right|^{2}}{2 \rho(\mathbf{r})}
$$

where

$$
\gamma\left(\mathbf{r}, \mathbf{r}^{\prime}\right)=2 \sum_{i} \phi^{*}(\mathbf{r}) \phi\left(\mathbf{r}^{\prime}\right),
$$

is the Dirac matrix. In the summation above, the index $i$ refers to quantum numbers other than the spin (summation over spin gives a factor of two that has been included explicitly in the expression above). Self-consistent calculations with $V_{\text {Slater }}(\mathbf{r})$ in place of $v_{\mathrm{xc}}(\mathbf{r})$ in (2) can be performed easily. The results (Harbola and Sahni 1993) obtained from such calculations for some closed-shell atoms are displayed in comparison with the Hartree-Fock numbers (Fischer 1977) in table 1. They amply demonstrate the accuracy of the Slater potential. The idea of Slater was further explored by Harbola and Sahni (1989) who recognized that the Slater potential was being calculated by treating the Fermi hole as a static charge distribution. However, the Fermi hole changes shape as a function of electron position in an inhomogeneous density. Therefore, the correct way to obtain the potential is to first get the electric field due to the Fermi hole and then calculate the potential as the work done in moving the electron in this field. Thus the correct exchange potential is given as

$$
W_{\mathrm{x}}(\mathbf{r})=-\int_{\infty}^{\mathbf{r}} \mathbf{E}_{\mathrm{x}}\left(\mathbf{r}^{\prime}\right) \cdot \mathrm{d} \mathbf{l}^{\prime},
$$

where

$$
\mathbf{E}_{\mathrm{x}}(\mathbf{r})=\int \frac{\rho_{\mathrm{x}}\left(\mathbf{r}, \mathbf{r}^{\prime}\right)}{\left|\mathbf{r}-\mathbf{r}^{\prime}\right|^{3}}\left(\mathbf{r}-\mathbf{r}^{\prime}\right) \mathrm{d} \mathbf{r}^{\prime} .
$$

is the electric field due to the Fermi hole. A comparison between the two potentials is made in figure 1 for the Argon atom. It is seen that the potential, $W_{\mathrm{x}}$, is shallower than the exact Slater potential in the interior of the atom and both go as $-\frac{1}{r}$ outside the atom. Comparison between the energies obtained from the two methods is made in table 1 . It is evident that both the total energies and the highest occupied orbital energies with $W_{\mathrm{x}}$ as the exchange potential come out to be closer to Hartree-Fock than the Slater potential. This indicates that the derivation of $W_{\mathrm{x}}$ invokes the right physics.

In the discussion above we have introduced the idea of a field due to the Fermi hole and have obtained the exchange potential as the work done in moving an electron in this field. However, this way of looking at the potential is absolutely general and applies equally well to the exchange-correlation potential. Thus the exchangecorrelation potential is (Harbola and Sahni 1989, 1990a, b) the work done in moving an electron in the field of its Fermi-Coulomb hole plus a small component representing the difference in the interacting and the non-interacting kinetic energy of a given density. The latter has to be added separately because Coulomb's law, on which the derivation of the potential is based, cannot take care of the kinetic energy component of the Kohn-Sham potential.

The physics of obtaining the exchange-correlation potential outlined above can be derived, as was shown later by Holas and March (1995), on the basis of the differential virial theorem. Accordingly, the gradient of the exchange-correlation potential is given as

$$
-\nabla v_{\mathrm{xc}}(\mathbf{r})=\frac{\mathbf{z}_{1 \mathrm{~s}}(\mathbf{r})-\mathbf{z}_{1}(\mathbf{r})}{\rho(\mathbf{r})}+\int \frac{\boldsymbol{\rho}_{\mathrm{xc}}\left(\mathbf{r}, \mathbf{r}^{\prime}\right)}{\left|\mathbf{r}-\mathbf{r}^{\prime}\right|^{3}}\left(\mathbf{r}-\mathbf{r}^{\prime}\right) \mathrm{d} \mathbf{r}^{\prime},
$$

where $\mathbf{z}_{1 \mathrm{~s}}(\mathbf{r})$ and $\mathbf{z}_{1}(\mathbf{r})$ are quantities related to the kineticenergy density tensor derived from the first-order KohnSham and true density matrices, respectively. Their difference represents the kinetic energy component of the Kohn-Sham potential. We point out that the individual components of the potential gradients could have a nonvanishing curl but their sum is always curl-free. Thus if one wants to calculate only the exchange-component of the potential, certain approximations, such as the centralfield approximation in the case of atoms, have to be made

\begin{tabular}{|c|c|c|c|c|c|c|}
\hline \multirow[b]{2}{*}{ Atom } & \multicolumn{3}{|c|}{ - Total energy (Hartree) } & \multicolumn{3}{|c|}{$-\varepsilon_{\max }($ Ryd.) } \\
\hline & Slater & $W_{\mathrm{x}}$ & $\mathrm{HF}$ & Slater & $W_{\mathrm{x}}$ & $\mathrm{HF}$ \\
\hline $\mathrm{He}$ & $2 \cdot 862$ & $2 \cdot 862$ & $2 \cdot 862$ & 1.836 & $1 \cdot 836$ & 1.836 \\
\hline $\mathrm{Be}$ & $14 \cdot 561$ & $14 \cdot 571$ & 14.573 & 0.653 & 0.626 & 0.619 \\
\hline $\mathrm{Ne}$ & $128 \cdot 501$ & $128 \cdot 542$ & $128 \cdot 547$ & $1 \cdot 824$ & 1.713 & 1.701 \\
\hline $\mathrm{Mg}$ & $199 \cdot 533$ & $199 \cdot 606$ & $199 \cdot 615$ & 0.568 & 0.521 & 0.506 \\
\hline $\mathrm{Ar}$ & $526 \cdot 703$ & $526 \cdot 804$ & $526 \cdot 818$ & $1 \cdot 276$ & $1 \cdot 178$ & $1 \cdot 182$ \\
\hline $\mathrm{Ca}$ & $676 \cdot 606$ & $676 \cdot 743$ & $676 \cdot 758$ & $0 \cdot 449$ & $0 \cdot 402$ & 0.391 \\
\hline
\end{tabular}

Table 1. A comparison of the total energies and highest-occupied orbital energies as obtained by employing the Slater (12) and the $W_{\mathrm{x}}$ (15) exchange potential in the Kohn-Sham equation. The numbers are compared with the corresponding HartreeFock (HF) theory results. Negative of the total energy and the orbital energy are given. 
(Sahni 1997). Note that self-consistent calculations with the potential being calculated above are possible only at the exchange-only level since only the exchange potential can be calculated directly from the orbitals by employing (15) and (16). On the other hand, the prescription above has been employed a great deal to study the properties of the exact Kohn-Sham potential. We also note that the arguments employed to derive the potential are equally valid for the excited-states and as such a local potential for the excited state can also be derived (Harbola and Sahni 1989; Sahni et al 2001) from the field arising from the Fermi-Coulomb hole. Excited-state energy calculations (Sen 1992; Singh and Deb 1999) based on this potential lead to highly accurate excitation energies.

Equation (17) above gives a way of constructing the exact exchange-correlation potential, which is coventionally obtained as the functional derivative of the exchange-correlation energy functional. Derivation of the potential as the functional derivative is based on the minimization of the energy whereas the equation above is derived directly from the Schrödinger equation. The two methods, of course, lead to the same results.

We now use the physics discussed above to derive an approximate exchange potential in terms of the exchange energy density. As described earlier, such an approach has the advantage of obviating the need to take functional derivatives of an exchange energy functional so that any error associated with that process is minimized.

\section{Exchange potential in terms of the exchange energy density}

To derive (Harbola and Sen 2002) the exchange potential in terms of the exchange energy density, we rewrite the electric field (16) due to the Fermi hole as

$$
\mathbf{E}_{\mathrm{x}}(\mathbf{r})=-\nabla V_{\text {Slater }}(\mathbf{r})+\int \frac{\nabla \boldsymbol{\rho}_{\mathrm{x}}\left(\mathbf{r}, \mathbf{r}^{\prime}\right)}{\left|\mathbf{r}-\mathbf{r}^{\prime}\right|} \mathrm{d} \mathbf{r}^{\prime},
$$

where $V_{\text {Slater }}(\mathbf{r})$ is the exact Slater potential as given by (12). We now approximate the second term by considering the gradient expansion of the Fermi hole up to the first-order in the gradient of the density. This gives (Wang et al 1990)

$$
\int \frac{\nabla \rho_{\mathrm{x}}\left(\mathbf{r}, \mathbf{r}^{\prime}\right)}{\left|\mathbf{r}-\mathbf{r}^{\prime}\right|} \mathrm{d} \mathbf{r}^{\prime}=-\nabla \frac{k_{\mathrm{F}}(\mathbf{r})}{2 \pi} .
$$

Now recognizing that the Slater potential is nothing but twice the exchange energy, $\varepsilon_{x}(\mathbf{r})$ per particle, we obtain an approximate exchange potential

$$
v_{\mathrm{x}}(\mathbf{r})=2 \varepsilon_{\mathrm{x}}(\mathbf{r})+\frac{k_{\mathrm{F}}(\mathbf{r})}{2 \pi} .
$$

The expression above is exact in the limit of homogeneous electron gas as it leads to $v_{\mathrm{x}}=-\frac{k_{\mathrm{F}}}{\pi}$. For inhomoge- neous systems we expect the second term on the right hand side of (20) to be different from $-\frac{k_{\mathrm{F}}}{\pi}$ so we parametrize the potential as

$$
v_{\mathrm{x}}(\mathbf{r})=2 \varepsilon_{\mathrm{x}}(\mathbf{r})+\frac{k_{\mathrm{F}}(\mathbf{r})}{\beta \pi},
$$

with $\beta$ as a parameter. For a given approximate $\varepsilon_{x}$, the parameter $\beta$ is fixed by minimizing the energy functional

$$
\begin{aligned}
E[\boldsymbol{\rho}]= & T_{\mathrm{s}}[\boldsymbol{\rho}]+\int v_{\mathrm{ext}}(\mathbf{r}) \boldsymbol{\rho}(\mathbf{r}) \mathrm{d} \mathbf{r} \\
& +\frac{1}{2} \iint \frac{\dot{\boldsymbol{\rho}}(\mathbf{r}) \boldsymbol{\rho}\left(\mathbf{r}^{\prime}\right)}{\left|\mathbf{r}-\mathbf{r}^{\prime}\right|} \mathrm{d} \mathbf{r} \mathrm{d} \mathbf{r}^{\prime}+\int \varepsilon_{\mathrm{x}}(\mathbf{r}) \boldsymbol{\rho}(\mathbf{r}) \mathrm{d} \mathbf{r},
\end{aligned}
$$

with respect to it. For example, if we use the Becke88 exchange energy functional, in which $\varepsilon_{\mathrm{X}}$ (form appropriate for spin-unpolarized systems is written here) is given as

$$
\varepsilon_{\mathrm{x}}(\mathbf{r})=-\frac{3 k_{\mathrm{F}}(\mathbf{r})}{4 \pi}-\gamma \sum_{\sigma} \int \rho_{\sigma}^{\frac{1}{3}} \frac{x_{\sigma}^{2}}{\left(1+6 \gamma x_{\sigma} \sin h^{-1} x_{\sigma}\right)},
$$

where $\sigma$ indicates the up or the down spin, $x_{\sigma}=\frac{|\nabla \rho \sigma|}{\rho_{\sigma}^{4 / 3}}$ and $\gamma=0.0042$, we find that $\beta=2 \cdot 5,2 \cdot 3$, and $2 \cdot 0$, and for $\mathrm{Ne}, \mathrm{Ar}$, and $\mathrm{Xe}$, respectively. It is gratifying to note that the minimizing $\beta$ is close to 2 and converges to this value for large systems, as expected.

We point out that minimizing energy with respect to $\beta$ is like searching for a minimum with a restricted set of densities. Thus the energy obtained by us would lie above that given by the densities corresponding to the functional derivative potential, since the latter is equivalent to minimization with respect to all possible densities.

We now present the results of self-consistent calculations for some closed-shell atoms by employing the expression of (21) for the exchange potential in conjunction with the Becke88 exchange energy functional given above. In the results reported, we have not minimized the total energy with respect to $\beta$ for each system but have taken an average fixed value of $\beta=2 \cdot 25$. Before presenting the numbers obtained by us, we note that since the Becke88 exchange-energy per particle goes as $-\frac{1}{2 r}$ for asymptotic distances from the nucleus, the potential in these regions as given by (21) goes correctly as $-\frac{1}{r}$. On the other hand, asymptotically the functional derivative of the Becke88 functional goes (Engel et al 1992) as $-\frac{1}{2^{2}}$. As such we may expect that the highest occupied orbital energies as obtained by us will be better than those given by the functional derivative of the Becke88 functional. In table 2 we compare our results with those obtained by solving the Kohn-Sham equation with the exchange potential calculated as the functional derivative of the Becke88 functional, and also with the Hartree-Fock results. It is evident from the numbers presented that the potential of (21) leads to accurate energies that are only 
Table 2. A comparison of the total energies and highest-occupied orbital energies as obtained by employing the Becke88 (B88) potential and the potential derived from (21) (PW) in the Kohn-Sham equation. The numbers are compared with the corresponding Hartree-Fock (HF) theory results. Negative of the total energy and the orbital energy are given.

\begin{tabular}{|c|c|c|c|c|c|c|}
\hline \multirow[b]{2}{*}{ Atom } & \multicolumn{3}{|c|}{ - Total energy (Hartree) } & \multicolumn{3}{|c|}{$-\varepsilon_{\max }($ Ryd.) } \\
\hline & B88 & PW & $\mathrm{HF}$ & B88 & PW & $\mathrm{HF}$ \\
\hline $\mathrm{He}$ & $2 \cdot 863$ & $2 \cdot 862$ & $2 \cdot 862$ & $1 \cdot 108$ & $1 \cdot 374$ & 1.836 \\
\hline $\mathrm{Be}$ & $14 \cdot 566$ & 14.563 & 14.573 & $0 \cdot 362$ & $0 \cdot 482$ & 0.619 \\
\hline $\mathrm{Ne}$ & $128 \cdot 590$ & $128 \cdot 586$ & 128.547 & $0 \cdot 909$ & $1 \cdot 230$ & 1.701 \\
\hline $\mathrm{Mg}$ & $199 \cdot 632$ & $199 \cdot 626$ & $199 \cdot 615$ & $0 \cdot 298$ & 0.422 & 0.506 \\
\hline $\mathrm{Ar}$ & $526 \cdot 800$ & $526 \cdot 792$ & $526 \cdot 818$ & 0.684 & $0 \cdot 910$ & $1 \cdot 182$ \\
\hline $\mathrm{Ca}$ & $676 \cdot 753$ & $676 \cdot 745$ & 676.758 & $0 \cdot 231$ & $0 \cdot 340$ & 0.391 \\
\hline
\end{tabular}

parts-per-million different from those obtained by employing the functional derivative of the Becke88 functional in the Kohn-Sham equation. Note that the energies obtained by us lie above those given by the functional derivative potential. This is expected as was pointed out above. On the other hand, since (21) gives the asymptotic behaviour of the potential more accurately, the highest occupied orbital energies obtained by us are substantially better: they are larger in magnitude than the corresponding Becke88 orbital energies, and closer to their Hartree-Fock values. Proximity of the two sets of numbers to the Hartree-Fock theory numbers points to the correctness of the approach taken by us.

In figure 1, we have also plotted the self-consistent exchange potential of (21) and the Becke88 functional derivative along with the Slater and the $W_{\mathrm{x}}$ exchange potentials. It is evident that the Becke 88 and the potential of (21) are very close to each other and also to $W_{\mathrm{x}}$ from about 0.03 a.u. onwards. However, near the nucleus, the Becke88 potential goes to $-\infty$ whereas the potential of (21) is finite. Further, it is closer to the $W_{\mathrm{x}}$ potential than the Slater potential although it also is slightly deeper than the $W_{\mathrm{x}}$ potential. Far from the nucleus the difference between the Becke88 potential $\left(-\frac{1}{2}\right)$ and the potential of (21) $\left(-\frac{1}{r}\right)$, is not apparent in the figure because of the scale used.

One quantity that brings out the difference in the behaviour of the potentials near the nucleus rather clearly is the cusp condition satisfied by the density. According to this condition, the ground-state density of a neutral atom satisfies (see, for example, Nagy and Sen 2001) the relationship

$$
\left.\frac{1 \mathrm{~d} \rho}{2 \rho \mathrm{d} r}\right|_{r=0}=-Z,
$$

where $Z$ is the atomic number. In the calculations employing the functional-derivative of the Becke88 functional, the left-hand side of (24) gives -2.04, -4.04, -10.04, $-12.04,-18.04$ and -20.04 , respectively, for $\mathrm{He}, \mathrm{Be}$,

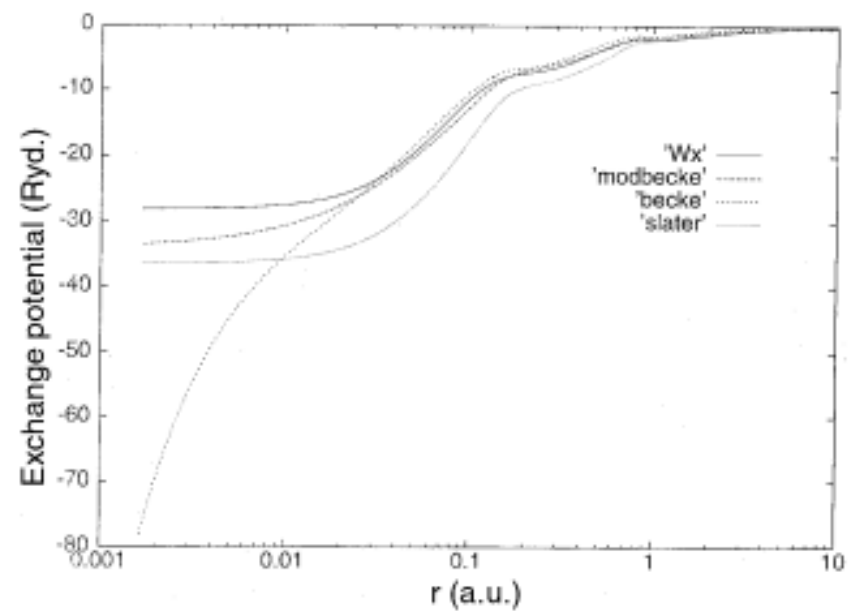

Figure 1. Different exchange potentials, $W_{\mathrm{x}}(15)$, modbecke (21), Becke (functional derivative of the Becke88 functional of (23)), and the Slater potential (12), for the Argon atom in Rydberg units.

$\mathrm{Ne}, \mathrm{Mg}$, Ar and $\mathrm{Ca}$. On the other hand, the potential of (21) leads to the correct cusp condition being satisfied for all these systems.

\section{Conclusions}

We have presented an approach to obtaining the KohnSham exchange potential directly from the exchangeenergy per particle, obviating the need to calculate the functional derivative of the corresponding functional. We have employed the method proposed in conjunction with the Becke88 functional and shown that our method not only gives the potential close to the functional derivative of the functional but also improves its behaviour significantly for all values of electron positions. Thus in addition to the energies being in agreement with those given by the potential derived as the functional derivative, the highest-occupied orbital energies obtained by our method are superior to the latter. Further, the densities obtained also satisfy the correct cusp condition. This indicates the 
correctness of our approach. We are now employing the potential derived to perform calculations on atomic anions and molecules by including correlation effects through the LYP functional. We are also planning to include higher order correction to the potential derived, although it is difficult due to the electric field from the third-order correction to the LDA hole having (Wang et al 1990) a non-vanishing longitudinal component. Investigations in this direction are in progress.

In the past also, attempts have been made to correct the LDA potential, particularly to improve its asymptotic behaviour. One of these corrections was introduced in the context of the $X \alpha$ potential by Latter (1955) and is known as the Latter correction. More recently, van-Leeuwen and Baerends (LB) (1994) added a correction, which is based on the Becke88 functional, to the LDA to make it asymptotically accurate. The numbers obtained by the LB potential show a vast improvement over the LDA numbers. However, the derivation by LB is adhoc. Gritsenko et al (1995) have proposed a partitioning of the exchange potential into a long range Slater and a short range response component. By replacing the Slater component with the GGA energy density and modelling the response component, their scheme also yields reasonable accurate representation of the optimized potential. Our derivation places such partitioning of the potential on a rigorous analysis, based on the physics of the Fermi hole, which can be systematically improved by including higher-order corrections. Further work on performing self-consistent calculations with correlation potential added to the potential above is also in progress and will be reported in the future.

\section{References}

Becke A D 1988 Phys. Rev. A38 3098
Dreizler R M and Gross E K U 1990 Density-functional theory (Berlin: Springer-Verlag)

Engel E, Chevary J A, Macdonald L D and Vosko S H 1992 Z. Phys. D23 7

Fischer C F 1977 The Hartree-Fock method for atoms (New York: Wiley)

Gritsenko O, van Leeuwen R, van Lenthe E and Baerends E J 1995 Phys. Rev. A49 1944

Gunnarsson O and Lundquist B I 1976 Phys. Rev. B13 4274

Harbola M K and Sahni V 1989 Phys. Rev. Lett. 62489

Harbola M K and Sahni V 1990a Int. J. Quantum Chem. Symp. 24569

Harbola M K and Sahni V 1990b Phys. Rev. Lett. 652609

Harbola M K and Sahni V 1993 J. Chem. Ed. 70920

Harbola M K and Sen K D 2002 Int. J. Quantum Chem. 89491

Herman F, van Dyke J P and Ortenberger I B 1969 Phys. Rev. Lett. 22807

Holas A and March N H 1995 Phys. Rev. A51 2040

Latter R 1955 Phys. Rev. 99510

Nagy A and Sen K D 2001 J. Chem. Phys. 1156300

Parr R G and Yang W 1989 Density-functional theory of atoms and molecules (New York: Oxford University Press)

Perdew J P 1985 Phys. Rev. Lett. 55 1665, 2370(E)

Perdew J P and Wang Y 1986 Phys. Rev. B33 8800

Perdew J P 1991 in Electronic structure of solids (eds) P Ziesche and H Eschrig (Berlin: Akademic-Verlag)

Qian Z and Sahni V 2000 Int. J. Quantum Chem. 79205

Sahni V 1997 Phys. Rev. A55 1846

Sahni V, Massa L, Singh R and Slamet M 2001 Phys. Rev. Lett. 87113002

Sen K D 1992 Chem. Phys. Lett. 188510

Singh R and Deb B M 1999 Phys. Rep. 311477

Slater J C 1951 Phys. Rev. 81385

Slater J C 1960 Quantum theory of atomic structure (New York: McGraw Hill) Vol. II

Umrigar C J and Gonze X 1994 Phys. Rev. A50 3827

van-Leeuwen R and Baerends E J 1994 Phys. Rev. A49 2421

Wang Y, Perdew J P, Chevary J A, Macdonald L D and Vosko S H 1990 Phys. Rev. A41 78 